\title{
Análisis crítico a las prácticas jurídicas de los estudiantes de derecho: El litigio estratégico como práctica alternativa e instrumento reivindicatorio de derechos sociales en Colombia'
}

\author{
Jeisson Fabian Porras Moreno²
}

jeissonporras@hotmail.com

Artículo de reflexión recibido el 26/04/2018

y aprobado el 11/07/2018

Cómo citar este artículo:

Porras-Moreno, J. (2018). Análisis crítico a las prácticas jurídicas de los estudiantes de derecho: el litigio estratégico como práctica alternativa e instrumento reivindicatorio de derechos sociales en Colombia. Trans-Pasando Fronteras, (12).

\footnotetext{
${ }^{1}$ Artículo de reflexión teórica nacida de la experiencia obtenida como monitor del Consultorio Jurídico de la Universidad Santo Tomas seccional Bucaramanga durante el año 2016-2017, en el marco de la realización de la judicatura como requisito para la obtención del título de abogado.

${ }^{2}$ Estudiante de Derecho de la Universidad Santo Tomas seccional Bucaramanga
} 


\section{Resumen}

El Derecho ha estado inmiscuido en situaciones que han comprometido su dimensión ética y su función social, esto ha llevado a que diferentes instituciones gubernamentales y académicas evalúen la necesidad de discutir y reformar las estrategias pedagógicas en las facultades de Derecho con el fin de mitigar las causas que dan origen a estas situaciones. Dentro de estos escenarios se ha concluido que uno de los factores a trabajar deben ser las mismas prácticas de los estudiantes, las cuales se ven mayormente reflejadas en el consultorio jurídico, ya que las mismas son el escenario propicio para reforzar las competencias éticas y la visualización del mismo como una ciencia social.

A raíz de esto, el objetivo de este trabajo es analizar de forma crítica como se aborda la concepción de la educación y la práctica jurídica para los estudiantes de derecho por parte del Estado y algunas universidades del país. Por otro lado, luego brindar el análisis mencionado, se expondrá la concepción del litigio estratégico como una forma alternativa de práctica jurídica y como propuesta de responder a las necesidades sociales en materia reivindicación y aplicación de derechos a las comunidades vulnerables desde la academia. Lo anterior tomando como referencia los postulados de la pedagogía crítica latinoamericana enmarcada dentro de la función social del abogado y de la universidad como institución social.

Con esto se busca aportar a esta discusión suscitada desde los diferentes estamentos sociales en referencia al Derecho y su necesidad de reformar sus estrategias pedagógicas, por lo que la alternativa de implementar los postulados del litigio estratégico puede brindar herramientas que permitan al estudiante desempeñarse en el área del conocimiento con la que más sienta afinidad y trabajar mano a mano con y para la comunidad que lo rodea.

Palabras clave: Litigio estratégico; Pedagogía crítica; Prácticas jurídicas; Estudiantes de derecho; Reivindicación de derechos. 


\section{Critical Analysis of the legal practices of the students of law: the strategic litigation as an alternative practice and the revindicating instrument of social right in Colombia}

\section{Abstract}

The Law has been intruder in situations that have compromised its ethical dimension and its social function, this has led to different governmental and academic institutions evaluate the need to discuss and reform the pedagogical strategies in law schools with the final porpuse to mitigate the causes that give rise to these situations. Within these scenarios it has been concluded that one of the factors to work should be the students' own practices, which are mostly reflected in the legal office, since they are the appropriate scenario to reinforce ethical competences and visualization of it as a social science.

As a result of this, the objective of this paper is to critically analyze how the conception of education and legal practice is addressed to law students by the State and some universities in the country. On the other hand, after providing the mentioned analysis, the concept of strategic litigation will be presented as an alternative form of legal practice and as a proposal to respond to social needs in terms of the claim and application of rights to vulnerable communities from the academy. The above taking as reference the postulates of Latin American critical pedagogy framed within the social function of the lawyer and the university as a social institution. 
This seeks to contribute to this discussion raised from the different social classes in reference to the law and its need to reform their pedagogical strategies, so the alternative of implementing the postulates of strategic litigation can provide tools that allow the student to perform in the area of knowledge with which you feel more affinity and work hand in hand with and for the community that surrounds you.

Key words: Strategic litigation; Critical pedagogy; Legal practices; Law students; Vindication of rights. 


\section{Metodología}

Para la realización de este trabajo se analizará en primera medida aspectos generales de la educación jurídica y la incorporación de las prácticas jurídicas en los planes educativos de los estudiantes de Derecho en Colombia; posteriormente se expondrán algunas de las propuestas del Estado y parte del sector universitario para las prácticas de los estudiantes de Derecho.

La segunda parte de este trabajo buscara dar una crítica a la ciencia jurídica desde dos aspectos: el primero desde un análisis al proyecto ilustrado y como la concepción de ciencia que se maneja al interior de las facultades de Derecho responde a este proyecto, generando en los estudiantes un alejamiento de la sociedad de la que hacen parte, con el fin de ser "observadores neutrales de los fenómenos sociales"3; el segundo aspecto se centrará a brindar una crítica al Derecho y como este, desde su actividad, busca la reafirmación de un poder establecido, lo que genera la necesidad de buscar una alternativa de una emancipación social y política. Como alternativa para este fin, se propondrá el litigio estratégico, teniendo en cuenta que uno de sus pilares es el trabajo con las comunidades socialmente marginadas y busca, a su vez, incentivar la creación de una conciencia social en los estudiantes de Derecho. Lo anterior desde el enfoque planteado por los postulados de la pedagogía crítica latinoamericana en la construcción de ciudadanías democráticas y críticas, siendo esta la última parte de este trabajo, la cual dará paso a las conclusiones de esta reflexión teórica.

${ }^{3}$ Esta expresión es empleada por Santiago Castro-Gómez en el 2010 en su libro "La hybris del punto cero: ciencia, raza e ilustración en la Nueva Granada (1750-1816)" y se desarrollará a mayor profundidad más adelante. 


\section{Introducción}

El conflicto armado en Colombia ha generado una serie de situaciones que han sido violatorias de derechos y garantías fundamentales para diferentes comunidades, sumado a problemas como la corrupción, la cual ha desencadenado la no generación de estrategias que permitan reivindicar derechos o a mitigar problemáticas. Por esto, tras la firma del acuerdo de paz firmado entre el Estado y las Fuerzas Armadas Revolucionarias de Colombia - Ejército del Pueblo (FARC-EP) en noviembre de 2016, diferentes organizaciones sociales han indagado sobre la necesidad de alcanzar una paz que vaya más allá que la cesación de enfrentamientos entre fuerzas irregulares y la fuerza pública, razón por la cual se han plateado diferentes temas para la reflexión tales como: la corrupción en la sociedad, el cuidado del medio ambiente, la reivindicación de derechos a la comunidades vulnerables, participación política de todos los sectores sociales, la formación de profesionales en una sociedad que demanda transformación social, crecimiento económico, ética en las actuaciones y pensamiento crítico; reconocimiento de las diferentes manifestaciones culturales, entre otros.

Por esta razón, replantear el papel de los estudiantes de las facultades de Derecho en una sociedad que demanda perspectivas críticas y agentes sociales activos de cambio, es una reflexión importante en este momento del país. Por tal motivo, tanto el Estado, a través del Ministerio de Justicia y del Derecho, la academia y diferentes organizaciones sociales, se han planteado diversas estrategias para inculcar en los estudiantes de Derecho una conciencia social, donde se les recalce el papel que toma el ejercicio de la profesión dentro de la comunidad en la que la ejercen. Un ejemplo para responder a esta necesidad de replanteamiento de la formación jurídica de los estudiantes de Derecho ha sido el Proyecto de Ley del año 2017 que 
modifica las competencias de los Consultorios Jurídicos o diferentes manifestaciones académicas como el texto lanzado por ACOFADE titulado: "Lineamientos técnicos para los programas de Derecho en Colombia". Estas dos manifestaciones guardan una similitud entre ellas, la necesidad de replantear las practicas jurídicas que tienen los estudiantes a lo largo de toda la carrera, en especial, de las realizadas en el Consultorio Jurídico los últimos semestres de su formación. Por ello es necesario hacer un ejercicio de análisis crítico de estas propuestas y a su vez plantear escenarios de participación de los estudiantes de Derecho, en medio de sus prácticas, con el fin de contribuir a la reivindicación los derechos de las comunidades vulnerables como una forma de aportar a la construcción de paz que demanda la sociedad colombiana.

Por lo anterior, la finalidad del presente trabajo de reflexión teórica, es plantear un análisis crítico sobre el estado actual de las practicas jurídicas de los estudiantes de Derecho, para luego establecer, a través de la figura de Litigio Estratégico, una alternativa de pedagogía critica que contribuya a la reedificación social por intermedio de la reivindicación de los derechos de las comunidades vulnerables.

\section{Antecedentes históricos de la educación jurídica en Colombia}

El desarrollo de la educación jurídica en Colombia ha tenido varias etapas a lo largo de su historia contemporánea. Por lo anterior, resulta de vital importancia determinar los cambios y circunstancias que acompañaron las diferentes reformas y estrategias pedagógicas en los estudiantes de Derecho y así poder determinar el momento en que se comenzó a desarrollar la idea de las prácticas jurídicas como componente formativo y social de los futuros abogados en Colombia. 


\section{Década de 1950}

Desde el año 1945 en Colombia comienza a nacer la necesidad de replantear y establecer ciertas críticas al papel de la educación en la sociedad, por tal motivo, y con el fin de ampliar las coberturas en la educación superior, se empiezan a realizar obras en materia de infraestructura, tales como la ciudad universitaria y se aumenta en un 63\% el cupo para acceder a las facultades; estas obras mencionadas se realizaron hasta el año 1954. Desde el plano de la reflexión, se planteó un problema frente al distanciamiento de la educación de la sociedad colombiana, esto, según varios sectores académicos, por la copia de los currículos de las universidades europeas, sin tener en cuenta las demandas de la sociedad colombiana (Molina y González, 2017). En el año 1948, tras la muerte de Jorge Eliecer Gaitán, liberales y conservadores inician un enfrentamiento armado y político, aspecto que hace entrar al país en un periodo de violencia física y estructural. Esta situación se generó una crítica a las universidades, ya que como en años anteriores, marcaban un aislamiento significativo al fenómeno de la violencia (Helg, 1984). Este tipo de críticas fueron también reforzadas por Alberto Lleras Camargo en 1954, donde manifestaba que una de las principales causas de la violencia era la falta de educación, pues esta acabo con la sensibilidad, así que la clave de tratar este problema de violencia estaba en mejorar el acceso a la educación y replantear las metodologías de la misma (Ramírez y Téllez, 2006).

A su vez, desde diferentes sectores políticos se enmarco una crítica a las universidades públicas por promulgar un pensamiento, aparentemente, comunista; un ejemplo de estas críticas a las universidades públicas, fue la expresada por el ex presidente Laureano Gómez, quien manifestó en la Universidad Javeriana, que la violencia que estaba sucediendo en el país se debía a un ataque de 
los enemigos de la iglesia cristiana, por lo que se toma una postura de rechazo frente a ideologías diferentes a las conservadoras y se categoriza al partido liberal también como comunista (Helg, 1984).

A raíz de este tipo de manifestaciones políticas de la iglesia y del gobierno de turno, se estimula la creación de instituciones universitarias de carácter privado y con carácter religioso, las cuales, tenían las libertades de tener proyectos y planes de estudio por fuera de los estipulados por el Ministerio de Educación Nacional (Molina y González, 2017), pero de forma simultánea, tras el exilio de profesores alemanes, franceses y españoles por lo acontecido en la Segunda Guerra Mundial, se genera la creación de nuevas facultades en el país que incentivan el uso de laboratorios y bibliotecas en la educación universitaria (Herrera, 1993).

Por lo anterior, es válido mencionar que esta década estuvo caracterizada por estar enmarcada en los primeros años de la época denominada como "la violencia"4, momento histórico que determino las dinámicas del conflicto armado contemporáneo. Dentro de estas dinámicas de la violencia bipartidista, se empezó a poner en el ámbito de la reflexión pública el papel de la educación como un motor de combatir y prevenir este problema, pero en medio de estas disertaciones, estaba también las manifestaciones del sector conservador colombiano, donde se estigmatizó las actividades de las universidades públicas, lo que incentivo la creación de universidades privadas con carácter religioso, dándoles una

4 Este periodo se comprendió entre los años 1948 y 1958, ubicando su inicio desde la muerte de Jorge Eliecer Gaitán hasta la creación del Frente Nacional. Esta época estuvo caracterizada por la ola de violencia entre conservadores y liberales, el golpe de Gustavo Rojas Pinilla y las dinámicas políticas que se generaron a raíz de lo mencionado anteriormente. Esta denominación fue brindada por Orlando Fals Borda, Monseñor Eduardo Umaña Luna y Germán Guzmán Campos en el libro "La violencia en Colombia” del año 1962. 
libertad de crear su propio modelo de formación, apartándose de los parámetros establecidos por el gobierno nacional. Simultáneamente la llegada de profesores extranjeros que huían de la segunda guerra mundial, incentivo a ampliar los campos de estudio en el país y a fortalecer la educación científica universitaria. Se puede inferir que, si bien hubo un sesgo marcado por la violencia, la cual estaba inmersa en el discurso de dirigentes universitarios y políticos de la década, se pudo generar escenarios que permitieron sentar las bases de la reflexión sobre la importancia de la educación en la solución de problemas neurálgicos que podía atravesar el país, tales como la violencia y la pobreza.

\section{Década de 1960}

En medio de la violencia, la educación, tal y como se había concebido en la década anterior, estaba en crisis. Por esta razón algunos críticos plantearon la necesidad de una reforma educativa donde se concibiera la enseñanza como acción instrumental, la cual tenía como fin buscar una mayor eficacia y un máximo rendimiento (Bocanegra, 2010).Por otro lado, el gobierno, a través de Decretos, comenzó a eliminar reglamentos en la educación para las facultades en Derecho, como la eliminación de la obligación de adherirse a los programas docentes de la Universidad Nacional y en 1966 se le otorga la autonomía a las universidades para elaborar sus propios planes de estudio (Gutierrez y Zarate, 2017). La crítica planteada en esta época trata de responder, por un lado, a una necesidad económica como lo fue la adaptación al sistema capitalista, tal cual y como se concibe actualmente a través de fenómenos como la globalización; sin embargo, a su vez, se dan los primeros pasos hacia concebir la autonomía universitaria por medio de la autonomía de elaboración de los planes de estudios de las universidades tras la 
reforma de 1966. No obstante, en sentido general, fue un periodo que trato de responder a las críticas de la década anterior, pero no hubo una actividad concreta en cuanto al papel del Derecho y la academia en la sociedad, sino un fortalecimiento al sector productivo en una naciente economía globalizada.

\section{Década de 1970}

Esta década se caracterizó por la inmersión directa de las actuaciones estatales en la formación de los abogados del país, como fue el caso de la promulgación del Decreto 196 de 1971, el cual reglamentó aspectos de la formación de los estudiantes de Derecho, así como la creación de la figura de los Consultorio Jurídicos, siendo este el primer contacto entre la academia y la sociedad. Esta inmersión fue significativa hasta el punto en que el estudiante adscrito al Consultorio Jurídico adquiere el rol de "abogado de pobres" otorgándole al mismo tiempo un rol dentro de la sociedad para velar por los intereses de las personas vulnerables en las materias determinadas por el mismo Decreto (Molina y González, 2017).

Durante estos años también apareció una preocupación por el ejercicio ético de la profesión del Derecho. Muestra de esto es que el Decreto citado anteriormente, también enmarcó reglas y parámetros éticos del ejercicio del abogado y del estudiante adscrito al Consultorio Jurídico como respuesta al desprestigio que la profesión del abogado estaba adquiriendo por el mal actuar de algunos profesionales. En este sentido, los parámetros, aparte de contener temas éticos, también tienen un enfoque dirigido a la importancia de la responsabilidad de las actuaciones (Molina y González, 2017).

${ }^{5}$ Esta expresión la contempla el mismo Decreto 196 de 1971 en su artículo 30 
Posteriormente se expidió el Decreto 3200 de 1979º el cual tenía la finalidad de establecer mayor control sobre la carrera del Derecho, enfatizando en dos aspectos claves, el ejercicio de la profesión y los contenidos mínimos de la formación del abogado. Dentro de estos parámetros mínimos se encontraba la inserción de la asignatura de ética profesional dentro de los planes de estudio de las universidades (Molina y González, 2017), esto buscando fortalecer y complementar lo estipulado con el Decreto 196 de 1971, ya que los estudiantes de Derecho ejercen la abogacía en circunstancias especiales. Estos antecedentes normativos fueron las primeras manifestaciones concretas acerca de la función del abogado y la materialización de la integración entre la academia y la sociedad, lo que permitió que en años posteriores se acuñaran expresiones como función social del abogado y la proyección social de las universidades.

\section{La década de 1980 y la actualidad}

En respuesta al naciente discurso ético en la profesión del abogado y también a la búsqueda que se propició con el fin de fortalecerlo, se dio expedición del Decreto 80 de 19807, el cual establece los principios de la formación profesional, dentro del cual se destaca la caracterización de la educación como un servicio público con función social, aspecto que permea la carrera de Derecho y fortalece lo establecido en los años 1971 y 1979 (Molina y González, 2017). Aunque la década de 1970 estableció parámetros normativos en cuanto a parámetros éticos y le otorgó una función social a la profesión del abogado, también se caracterizó por una crisis económica mundial, lo que conllevó a una crisis financiera y administrativa en la educación nacional, lo que

7 Derogado por la Ley 30 de 1992 
generó un grado de insatisfacción en la calidad de la educación y sus resultados. Esto incentivó que se expidiera la Ley 30 de 1992, con la cual organiza la educación superior como servicio público. Posteriormente, en el año 2000 se expide la Ley 583, la cual reforma algunos aspectos relacionados a la competencia de los Consultorios Jurídicos contemplado en el Decreto 196 de 1971, pero no lo derogó. En el año 2001 se expide el Decreto 2802, el cual estipuló los contenidos curriculares que deben contener las facultades de Derecho en el país, con el fin de alcanzar los estándares de calidad de la respectiva facultad. Con la promulgación de la Ley 1188 de 2008 se establecen el registro calificado de todos los programas universitarios del país (Huertas, 2017), la cual es reglamentada por el Decreto 1295 de 2010, que establece los registros calificados de las facultades universitarias (Molina y González, 2017).

Estas han sido las últimas manifestaciones normativas que ha habido en materia de formación profesional del abogado y de todas las carreras universitarias en general. Claramente tienen un enfoque más administrativo que formativo, pues nacen de la necesidad de reorganizar la educación superior luego de una crisis económica, pero además, mantiene la visión de la función social desarrollada en la década anterior, siendo la década de 1970 la encargada de marcar el paradigma normativo de la concepción de la función social del estudiante de Derecho y del mismo abogado,

8 Esta connotación se ve desarrollada en la Constitución Política de 1991 en su artículo 67, donde se estipula con una doble connotación, como derecho y como servicio público. En cuanto a la calidad de derecho, la educación tiene una incidencia en otros derechos como la escogencia de profesión u oficio, la dignidad humana, y además cuenta con la función de erradicar la pobreza y fomentar el desarrollo humano. La connotación de servicio público traslada al Estado a obligación de garantizar su eficiencia bajo los principios de solidaridad, eficacia y solidaridad, siendo esta ser prestada de forma continua y progresiva a todos los habitantes (Sentencia T-743, 2013) 
pero que a su vez, las demás manifestaciones radican es desde las iniciativas universitarias mediante la creación de sus planes de estudio y la configuración de sus prácticas, más allá de las plateadas por el Estado en año 1971. Resulta significativo como desde el año 1992 hasta el año 2008 no hubo algún tipo de acciones legislativa o ejecutiva en pro de la educación jurídica o universitaria en general, razón por la cual muchos sectores académicos han manifestado que existe un estancamiento en materia educativa, desde un punto de vista más sustancial, pues la regulación que ha habido en esta última etapa normativa de la educación universitaria se ha limitado más a establecer parámetros orgánicos y administrativos, que establecer un rol protagonista y significativo a los estudiantes en los procesos de transformación social del país.

\section{Elementos curriculares, la proyección social y la función social de la universidad en las facultades de Derecho en Colombia.}

En el acápite anterior se exploró de la evolución normativa que tuvo la educación jurídica en Colombia y cómo estas normas fueron regulando aspectos de la formación de los futuros abogados del país, hasta llegar a las concepciones actuales sobre los objetivos a perseguir por las universidades. A raíz de estas disposiciones normativas y administrativas, aparecen conceptos como mallas curriculares, la proyección social en las universidades y la función social de las mismas. Con el fin de poder determinar la visión de las facultades de Derecho en cuanto a su labor social, resulta fundamental trabajar estas concepciones y como las universidades del país, a nivel general, las desarrolla actualmente en pro de cumplir con esta exigencia legal y social. El primer concepto que se debe tratar es el de currículo, Marisa Cazares (2008) lo define como un camino de aprendizaje, el cual emana de una realidad 
histórica. Esto lleva a establecer una serie de elementos al proceso educativo, los cuales permiten responder a unos fines planificados anticipadamente, donde se reconoce al sujeto como ser político, económico, social e histórico. Por tal motivo se puede establecer que los currículos tienen como tarea fundamental alcanzar estos fines planificados con anticipación, por lo que se vale del uso de componentes didácticos donde se establezca una relación entre el hombre y el momento histórico en el que se encuentra dentro de su proceso de formación (Cazares, 2008).

Huertas (2017) manifiesta que los currículos en las facultades de Derecho en Colombia buscan que el estudiante adquiera competencias cognitivas, investigativas, interpretativas, argumentativas y comunicativas; y que a su vez adquiera capacidades en campos de la conciliación, el litigio y el trabajo interdisciplinario. Estas capacidades se obtienen con el ejercicio de los componentes y áreas que comprenden la actividad del abogado a lo largo de la formación de los estudiantes, tales como: "Área jurídica, área humanística, componente transversal de análisis lógico-conceptual, Interpretación constitucional y legal, argumentación jurídica y Prácticas profesionales" (Huertas, 2017, p. 79). Las prácticas profesiones se ven reflejadas en el curso de la asignatura de Consultorio Jurídico, la cual se ve en los últimos dos años de la carrera del estudiante, y con la cual el mismo estudiante entra en contacto directo con la sociedad.

De igual forma se puede decir que la misma asignatura es una forma de cumplir con las actividades de proyección social, la cual se puede definir como las acciones orientadas a formar y desarrollar en el estudiante un compromiso social mediante el acercamiento de este a la realidad para contribuir en la búsqueda de soluciones políticas y jurídicas a los fenómenos sociales que lo rodean (Huertas, 2017). 
Este ámbito de la proyección social al interior de las universidades busca cumplir con dos aspectos: cumplir con la función social de la educación y una forma de materializar el compromiso adquirido con la búsqueda del desarrollo de la sociedad (Huertas, 2017).

Otro aspecto que complementa este ámbito es la realización de investigación socio-jurídica al interior de las universidades que le permita al estudiante reconocer el contexto social y la problemática de los grupos sociales con la finalidad de generar respuestas, y así poder interiorizar estos conocimientos en los estudiantes antes de llegar a sus prácticas jurídicas en el Consultorio Jurídico (Carreño y González, 2017). La proyección social tiene como propósito traer de vuelta la concepción de responsabilidad social universitaria, esto mediante el cumplimiento los tres propósitos fundamentes que fundamenta todo su ejercicio: validar la docencia y la investigación con impacto social, generar conocimiento y difundir y transformar el entorno (Montoya, 2017).

Lo anterior puede indicar que el fin último de la proyección social como concepción es la búsqueda del cumplimiento de la responsabilidad social universitaria, la cual tiene como fin primordial la búsqueda del desarrollo humano (De la Calle y Jiménez, 2011), tomando como base sus capacidades funcionales como: integridad física, salud corporal, sentimientos, imaginación, pensamiento, emociones, razón práctica, individualidad, control político, derecho de asociación, libertad y cultura (Montoya, 2017). Permitiendo entender al ser humano como un ser complejo que en su cotidianidad desarrolla y ejecuta acciones que lo ayudan a trascender como miembro de una sociedad y como ser individual.

El estudio de la estructura que acompaña los planes de estudio de las facultades de Derecho permite establecer las finalidades y propósitos que se tiene como facultad y como universidad gen- 
eral. Como consecuencia de esto, las mallas curriculares permiten establecer todas las estrategias pedagógicas que acompañaran al estudiante de Derecho en toda su formación profesional; dentro de estas se puede encontrar la forma de ejecución de las prácticas jurídicas mediante los Consultorios Jurídicos y como su actividad tiene armonía con los parámetros de responsabilidad social universitaria que tiene la institución. Cabe aclarar que estos parámetros tienen un origen propio, en principio, ya que como lo menciona De la Calle y Jiménez (2011) estos emanan de las problemáticas propias que afronta la sociedad y la misión que adopta la universidad para afrontarlos. Actualmente la actuación que tiene el Consultorio Jurídico en materia de intervención social está limitada al ámbito litigioso. Lo anterior tiene fundamento en la concepción y el propósito con el que fue creada esta asignatura dentro de los programas de Derecho en el país mediante el Decreto 196 de 1971, ya que como menciona en sus competencias, el ejercicio del estudiante que cursa la materia está en la de intervenir en asuntos policivos, penales, civiles, administrativos y constitucionales ${ }^{9}$; siendo estas intervenciones meramente litigiosas y que no le permiten establecer un contacto directo con la comunidad en la que se encuentra y las problemáticas que la rodean como colectividad.

Por otro lado se encuentra que dentro del ámbito de la proyección social es la elaboración de investigaciones socio-jurídicas que tengan como finalidad la indagación de problemáticas en específico mediante la búsqueda de conocimiento teórico que permita

9 Frente a estas dos últimas competencias cabe aclarar que el estudiante en materia administrativa solo puede actuar en unos determinados procedimientos concretos como procesos disciplinarios y de responsabilidad fiscal como abogado de oficio, y en materia constitucional su actuar se limita a la protección de Derechos Fundamentales por intermedio de la acción de tutela o de velar por los intereses del usuario para acceder a la administración mediante la elaboración de quejas o derechos de petición. 
abordar la problemática desde ámbitos prácticos. Lo anterior no se encuentra articulado con la actividad del Consultorio Jurídico por mandato normativo pero en varias instituciones del país se está reflexionando la posibilidad de incluir este componente en la formación práctica del abogado y de abordar de forma más directa la problemática de colectividades junto a su práctica en la solución de conflictos interpartes.

\section{Visión contemporánea de los Consultorios Jurídicos y la inves- tigación socio-jurídica en Colombia}

Anteriormente se expuso que las universidades, mediante el accionar del Consultorio Jurídico y del incentivo del desarrollo de proyectos de investigación socio-jurídica, buscan cumplir a cabalidad con sus propósitos de proyección social, los cuales están dirigidos en cumplir con su postulado propio de responsabilidad social universitaria. No obstante, por lo general, estas actividades están desarticuladas, ya que las actividades prácticas del estudiante solo se enfocan en el componente litigioso y el aspecto investigativo queda relegado a ser desarrollado de forma alterna a sus prácticas o antes de entrar a estas. Siendo esta una problemática que tiene origen en la primigenia regulación que se le dio a la misma práctica.

Esta situación, aun y con las necesidades contemporáneas, no ha tenido una manifestación clara por parte de legislador ni del ejecutivo, de ahí que algunos académicos e instituciones hayan adoptado alternativas para articular el ejercicio investigativo en los Consultorios Jurídicos, con el fin de adoptar estrategias dirigidas a mitigar los problemas de comunidades vulnerables. Frente a la necesidad de ampliar los espectros de la actuación de los Consultorios Jurídicos y su inminente articulación con la actividad investigativa 
socio-jurídica. González y Duque manifiestan que el Derecho se desarrolla dentro de un espacio social, por ende, su conocimiento es aprehensible a la sociedad, razón por la cual se deben adoptar estrategias de comunicación e intervención social con el fin de humanizar el Derecho mismo. Frente a estas estrategias, las autores sugieren establecer ejercicios de prácticas o clínicas jurídicas y no limitar el estudio del Derecho a un mero aspecto de análisis dogmático-jurídico (Duque y González, 2008).

En este mismo aspecto coincide Castro, quien establece aplicar que el modelo clínico anglosajón en las prácticas jurídicas permite: establecer una relación horizontal entre el docente y el estudiante, incentiva la interdisciplinariedad, permite una formación de competencias investigativas, fomenta la oralidad y la escritura, dota de naturaleza estratégica a los casos y concientiza de la responsabilidad social de la profesión (Castro Buitrago, 2006)

Lo anterior se funda en el propósito y la finalidad que tiene el método de enseñanza clínica del Derecho, la cual:

"Propone unos parámetros de educación legal que distan de la enseñanza clásica del derecho, rompiendo el esquema de lo magistral para entrar en escenarios reales, de contacto con la práctica y apuesta por la aplicación de los conocimientos adquiridos en la carrera y, de otro lado, pone al estudiante de cara a su realidad, para mostrarle que a partir de sus conocimientos puede hacer grandes aportes para la solución de problemas reales, pero más allá de eso, para mostrarle la proyección social de su profesión y su responsabilidad con la sociedad en la cual está inmerso" (Castro, 2006, p. 179) 
Esta propuesta de dar aplicabilidad a este sistema de enseñanza del Derecho se funda en la necesidad de reivindicar la labor social del abogado, otorgando en los estudiantes la misión de defender el interés público, como una forma de ampliar o reformular su misión como futuro profesional (Londoño, 2016).

La necesidad de reformular la enseñanza del Derecho y la práctica del estudiante para que adquiera un sentido social marcado, va acompañada a otros problemas que sobrecogen el país, tales como la corrupción en el sistema judicial, el deterioro de la percepción ciudadana sobre el funcionamiento de la justicia y el papel de los abogados y la necesidad de establecer una revisión integral de la formación del abogado. (Molina y González, 2017).

Betancour y González (2017) exponen que uno de los resultados más contundentes de esta problemática es la sanción a aproximadamente a 14.000 abogados por faltas disciplinarias y ejercicios indebidos de la profesión por parte de los abogados. Para estos autores, la crítica que se hace al sistema educativo en lo correspondiente a las facultades de Derecho radica en la inactividad normativa en aspectos sustanciales en la formación de abogados; muestra de lo anterior es que el último parámetro sobre los aspectos mínimos que debe contener la formación de estudiantes de Derecho es la Resolución 2868 de 2003, lo que genera que el sistema jurídico no responda a las necesidades contemporáneas de la sociedad colombiana y el papel de la educación para responder a las mismas.

Según Molina y González (2017), esta inactividad normativa y falta de manifestación por parte del Estado, genera unas problemáticas educativas en las facultades de Derecho, como lo son: 
- Falta de regulación en competencias comunicativas, éticas, investigativas, cognitivas y conflictivas,

- Falta de elementos mínimos de exigencia en la formación actual en aspectos como la investigación, la escritura y la oralidad, políticas y técnicas pedagógicas que respondan a las necesidades contemporáneas de la sociedad colombiana; carencia de un entrenamiento práctico y de establecer énfasis en métodos alternativos como la enseñanza clínica del Derecho e incentivar la creación de laboratorios de práctica judicial, incentivo al uso de estrategias tecnológicas, dominio de lenguas extranjeras, establecer competencias interdisciplinarias, reforzar la formación ética y motivar la flexibilización de la formación;

- Falta de articulación entre los contenidos mínimos de calidad y las competencias básicas del aprendizaje; y

- Falta de una política pública dirigida a la formación de estudiantes y de forma especial a jueces y funcionarios.

Estas problemáticas expuestas están dirigidas a replantear los contenidos esenciales en la formación jurídica, tanto a estudiantes como profesionales y funcionarios públicos, pues la problemática social abarca tanto los problemas propios de cada comunidad vulnerable como del mismo acceso a la justicia. No hay que desconocer que las universidades han planteado reflexiones sobre las reformas educativas que se deben hacer en las facultades de Derecho, generando estrategias que puedan responder a las necesidades sociales. Estas se han amparado en la autonomía universitaria, ya que el Estado, hasta el 2016, no había expresado en alguna manifestación la necesidad de establecer una reforma al 
proceso educativo de los profesionales. Entre el 2016 y el 2017, el Ministerio de Justicia y del Derecho comenzó a entablar una serie de foros a nivel nacional, donde han socializado con diferentes universidades la idea de la reforma a los Consultorios Jurídicos. Según el Ministerio, uno de los motores y fines de esta reforma es:

\begin{abstract}
"Fortalecer los consultorios jurídicos y las prácticas jurídicas de las facultades de derecho. Estimamos necesario ampliar las competencias de los mismos y ofrecer un mayor rango de acción a los estudiantes, pero sobre todo, generar mejores y mayores espacios de acceso a la justicia para la población vulnerable" (Ministerio de Justicia y del Derecho, 2016)
\end{abstract}

Dentro de este Proyecto de Ley de reforma se puede ver toda una visión estructural del funcionamiento de los Consultorios Jurídicos, comenzando desde la misma definición que se contempla en el artículo 2:

"El Consultorio Jurídico es un escenario de aprendizaje práctico de las universidades, autorizado en los términos de esta ley, en el cual los estudiantes, bajo la supervisión, la guía y la coordinación del personal docente y administrativo que apoya el ejercicio académico, adquieren conocimientos y desarrollan competencias, habilidades y valores éticos para el ejercicio de la profesión de abogado, prestando el servicio obligatorio y gratuito de asistencia jurídica a la población establecida en la presente ley" (Ministerio de Justicia y del Derecho, 2017, p. 1) 10 .

10 El presente documento corresponde a la cuarta versión de la reforma de las competen-
cias de los consultorios jurídicos, el cual fue enviado a las diferentes universidades de la ciu-
dad de Bucaramanga para socializarlo el mes de Febrero del año 2017 en uno de los foros
organizados por el Ministerio de Justicia con el fin de comentarlo y aportar ideas al mismo.
Por tal motivo, en el desarrollo de la judicatura tuve acceso directo al documento por ser
funcionario del Consultorio Jurídico de la Universidad Santo Tomas seccional Bucaramanga. 
Evidenciando un cambio significativo con el Decreto 196 de 1971, donde no se enmarcaba un concepto claro a los Consultorios Jurídicos como institución y además relegaban al estudiante únicamente como "abogado de pobres", desconociendo las otras competencias que podía adquirir y de determinar a su vez un rol en la sociedad más contundente frente a su contribución a la transformación de la misma.

Es también relevante como la formación ética juega un papel preponderante en este proyecto, como necesidad de fortalecer este ámbito en los estudiantes; de igual forma se refuerza en el artículo 3 numeral 3 del mismo proyecto ${ }^{11}$ donde se enmarca la formación integral aspectos: académico, profesional, técnico, humano, social y ético, reconociendo que el ser humano, en medio de su actividad profesional, posee diversas dimensiones que motivan su actuar. Donde además se complementa en el artículo 4 con objetivos como la formación práctica ${ }^{12}$ como refuerzo de la formación teórica, fundamentándola con aspectos éticos y humanísticos; y la proyección social ${ }^{13}$ entendida a la realización de acciones que creen en el estudiante la conciencia de su función social dentro de la sociedad (Ministerio de Justicia y del Derecho,

11 Artículo 3: 3. Formación Integral. El Consultorio Jurídico constituye un escenario idóneo para la formación académica, profesional, técnica, humana, social y ética del abogado, permitiendo a los estudiantes de Derecho adquirir, mediante experiencias propias del ejercicio de la profesión, los saberes y habilidades necesarias y esperadas para el ejercicio de la abogacía. 12 Artículo 4: 1. Formación Práctica. Fortalecer el proceso educativo de los estudiantes de las facultades de Derecho mediante la articulación de la teoría y la práctica a partir de casos reales con un enfoque humanístico y ético.

13 Artículo 4: 3. Proyección social. Promover la apropiación de conciencia en los estudiantes de Derecho acerca de la función social que comporta el ejercicio de la abogacía, en cuyo desarrollo se debe actuar como agente activo en la reducción de la desigualdad y el alcance de la equidad social.

14 Artículo 3: 5. Función social. El Consultorio Jurídico orienta su acción a la defensa de derechos de sujetos de especial protección constitucional y personas naturales que carez- 
2017). Frente a la función social de los estudiantes de Derecho ${ }^{14}$ y en especial los del Consultorio Jurídico, se da un cambio de concepción de "abogado de pobres", dando al estudiante el deber de proteger a sujetos de especial protección constitucional, personas naturales que carezcan de medios económicos para contratar un abogado o grupos y personas en situaciones especiales que se encuentren en situaciones de vulnerabilidad o indefensión (Ministerio de Justicia y del Derecho, 2017).

También resulta importante evidenciar cómo la misma propuesta emanada del Ministerio de Justicia y del Derecho reconoce la importancia de articular los ejercicios investigativos a la práctica jurídica como forma de brindar respuestas a las problemáticas que acoge la comunidad en la que ejerce sus actividades . Esta relación se refuerza en el artículo 10 del Proyecto de Ley de reforma a los Consultorios Jurídicos, donde dentro de las competencias generales de los mismos, aparte de tener presente el componente litigioso, enmarca un actuar en "pedagogía en derechos y el ejercicio del litigio estratégico" (Ministerio de Justicia y del Derecho, 2017, p. 4), teniendo como población objeto las comunidades vulnerables y los sujetos de especial protección constitucional.

Resulta interesante cómo sectores académicos y estatales se han puesto de acuerdo en aspectos fundamentales en los procesos de formación jurídica como: la necesidad de una reforma

can de medios económicos para contratar los servicios de un profesional en Derecho, o en general personas o grupos que, por sus circunstancias especiales, se encuentren en situación de vulnerabilidad o indefensión.

${ }^{15}$ Artículo 4: 4. Innovación jurídica. Propiciar el conocimiento científico, reflexivo e innovador del Derecho, atendiendo a las realidades contemporáneas de interés para el campo jurídico y que tengan impacto sobre el contexto socioeconómico de las comunidades donde ostenta influencia la facultad de derecho. 
en la concepción de las prácticas jurídicas, el refuerzo de los parámetros éticos, la incersión de los procesos de investigación sociojurídica a los procesos prácticos, la importancia de la creación y el refuerzo de la función social que tienen los estudiantes de Derecho y los abogados en general.

Como alternativa clave para llevar a cabo estas reformas, se propone desde el ámbito académico realizar procesos de enseñanza clínica del Derecho como estrategia de adentrar a los estudiantes a la sociedad y las dinámicas en las que se desarrolla, donde también pueden realizar procesos investigativos dirigidos a la transformación del espectro social en que ejerce la universidad. Por otro lado, el Estado, desde el Proyecto de Ley del año 2017 por el cual se busca modificar las competencias de los Consultorios Jurídicos, propone como método de intervención en la sociedad, la implementación del litigio estratégico como eje central de las actuaciones del Consultorio Jurídico y su intervención en la comunidad.

Lo que se puede resaltar sobre esta propuesta del gobierno colombiano es que no da un parámetro guía sobre cómo actuar bajo los lineamientos del Litigio Estratégico, por lo que deja que cada institución lo implemente de la forma en que más le convenga trabajar, por lo que uno de los interrogantes surgidos de estas propuestas y manifestaciones de cambio en los procesos de la formación de los estudiantes de Derecho y sus prácticas, es si ¿Realmente resultan suficientes estas aproximaciones reflexivas para poder contribuir a las problemáticas de una sociedad tan diversa como la colombiana, que a su vez tiene problemáticas y características particulares dependiendo la región en que tenga actividad cada universidad del país? 


\section{Elementos críticos al estudio de la ciencia del Derecho y a su función organizadora de la sociedad}

Para responder el interrogante enunciado en el acápite anterior, es importante empezar señalando unos elementos que pretenden responder, desde un punto de vista crítico y reflexivo, las razones que permitieron que las prácticas y la educación jurídica, se cimentaran bajo la óptica de no inmiscuirse en las problemáticas sociales y solo tener como objeto de estudio la norma y las instituciones que la rodeaban.

El proyecto de la ilustración del Siglo XVIII que tuvo origen en Europa, tuvo una serie de procesos que le permitieron expandirse por todo el mundo. El primer proceso tuvo como base colonizar el lenguaje y hacer una distinción entre el lenguaje cotidiano y el lenguaje exclusivo que sería utilizado en la ciencia; esto permitió tener un metalenguaje universal de la ciencia, el cual tenía como finalidad tomar una distancia del lenguaje cotidiano, con el fin de observar desde un punto neutro todos los fenómenos sociales para poderlos estudiar (Cástro-Gómez, 2010).

Cástro-Gómez (2010) denomino a este punto neutro de observación y estudio de la sociedad como Hybris del Punto Cero, el cual generaba el lenguaje propio de la ciencia, que no tenía ubicación geográfica concreta al ser generada de un punto neutro de observación. Este uso del lenguaje dotó de una estructura universal a la razón.

Este cambio en los usos del lenguaje no fue un fenómeno aislado de la ilustración y su cambio de concepción en los estudios de las ciencias modernas y de las nacientes ciencias humanas, sino que además trajo consigo una serie de cambios sociales y de mentalidad que caracterizaron el Siglo XVIII. Dentro de los cambios sociales se pueden encontrar 3 momentos específicos: el desar- 
rollo de las ciencias, nuevos asentamientos de colonias europeas y la expansión de la economía capitalista. Lo anterior generó la transformación de la mirada del mundo desde el ámbito social y natural (Cástro-Gómez, 2010).

Dentro de los cambios de mentalidad originada de estos fenómenos sociales enunciados, Cástro-Gómez (2010) señala, alguno, dentro de los cuales se pueden destacar:

- Lógica y retorica: se establecieron aspectos tales como campos legítimos de la ciencia, y forma de transmisión legitima de saberes, la cual estaba basada en la demostración escrita y matemática mediante la aplicación de un método científico, el cual permitía validar el trabajo realizado.

- Teoría jurídica y moral: hubo un cambio de regulación del ser humano en cuanto a la moral, mutando este concepto al de ética desde un punto de vista kantiano, donde los seres humanos se regían bajo unos principios universales de comportamiento; con esto se pudo determinar un entendimiento y resolución de los casos particulares.

Con estos cambios de mentalidad mencionados anteriormente, permitió que la observación desde el punto cero estuviera muy ligada a las estructuras de poder, pues valiéndose del uso de la razón construyo, instruyo y represento una visión del mundo, la cual era legitimada por el Estado (Cástro-Gómez, 2010).

Esta fuerza dominadora del Estado se vio enmarcada en la generación de todo un aparato burocrático, el cual era definido por Marx como: 
"El "formalismo del Estado" de la sociedad civil. Es la conciencia del Estado, la voluntad del Estado, el poder del Estado como corporación, es decir una sociedad particular, cerrada en el Estado. (Frente al interés particular, el interés general no puede ser más que un particular, en tanto lo particular es, frente a lo general, un general. La burocracia está obligada, pues, a proteger la particularidad imaginaria del interés general, para proteger a la particularidad imaginaria del interés general, a su propio espíritu. El Estado debe ser corporación como mientras la corporación quiere ser Estado)" (Silva Rojas, Aguirre Román \& Maldonado, 2013, p. 101).

Lo que lleva a decir que todo la organización burocrático se convierte a su vez en un aparato de dominación y de difícil acceso para la sociedad, pues el Estado a través de la legitimación de todo el lenguaje de la ciencia fundamentó su actuar y la conformación de cierta institucionalidad que le brinde estabilidad al orden establecido; a tal punto que el Derecho es utilizado para crear y establecer estos parámetros de acceso y exigibilidad de los derechos (Silva, Aguirre y Maldonado, 2013). Esta clase de problemáticas era la que se manifestaba en la sociedad colombiana en la década de 1950 cuando se comenzó a generar la reflexión sobre el papel de la educación en la solución de los problemas y como la educación estaba alejada de todo rol social. Aunque el tiempo fue avanzando y las diferentes críticas al sistema educativo giraban en torno a mitigar la brecha que había entre los estudiantes y la sociedad, pero aun esta problemática está inmersa en la sociedad colombiana, por lo que a la actualidad se han debatido en diferentes espacios que hacer para garantizar un real acceso a la población vulnerable y poder determinar una lucha por la garantía de los derechos. Tal como lo menciona Silva et al: 
"De lo que se trata es de luchar por los derechos generales del hombre en cuanto al hombre, no en cuanto a ciudadano, ni en cuanto a burgués, ni en cuanto trabajador, etc., y de oponerse de manera radical a cualquier forma de opresión y sometimiento que viole su condición de ser humano" (Silva et al, 2013, p. 87).

Esta lucha por los derechos se debe fundamentar desde dos aspectos fundamentales: la compresión de sí mismo y del mundo que lo rodea (Silva et al, 2013); así como la toma de conciencia de la situación que rodea al estudiante de Derecho y de buscar alternativas de cambio. De ahí que la propuesta de este trabajo está encaminada a impulsar la figura del litigio estratégico como aspecto reivindicatorio de Derechos, donde los estudiantes sean los instrumentos para que las comunidades vulnerables puedan acceder de forma directa al Estado con el fin de reivindicar aquellas carencias que poseen.

Este actuar del litigio estratégico se fundamenta en que el objeto principal de esta técnica de práctica y enseñanza jurídica es el trabajo con las organizaciones sociales y las comunidades que requieren este acompañamiento de los estudiantes de Derecho (Bonilla, 2008), para esto te toma lo propuesto por Correa (2008), quien establece que el litigio estratégico se vale de tres componentes que aglutinan su esfera de acción:

- Componente Jurídico: con este componente se materializa el actuar del estudiante de Derecho, pues es la forma de poner en práctica lo aprendido durante toda su carrera mediante la intervención en asuntos propios de reivindicación de alguna comunidad. Esta puede ser a través del movimiento del aparato judicial o del acompañamiento en los procesos de creación de política pública. 
- Componente político: este componente es la motivación del estudiante para realizar el activismo en pro de la población vulnerable, pues es el establecer que su ejercicio estudiantil de intervención lo hace como un agente político y social de cambio.

- Componente comunicativo: una de las mayores problemáticas que se presentan en la sociedad en general se debe al desconocimiento sobre cómo acceder al Estado para exigir las garantías a las que se tiene derecho, por tal motivo, en este componente se establece este dialogo entre los estudiantes de Derecho y la comunidad en la que ejercen su función con el fin de establecer parámetros pedagógicos que puedan ser de utilidad para la comunidad a la hora de acudir al Estado.

La ejecución de estos componentes debe ser de manera armónica y sobre todo de transmitir en los estudiantes y de emplear conceptos como la construcción de ciudadanías propias, esta concepción de ciudadanía va encaminada a la construcción de la sociedad latinoamericana basada en la aceptación del otro y de su forma de conocer el mundo, donde se deje los sesgos culturales, raciales, religiosos y sociales. La invitación es aportar al cambio desde la concientización al estudiante de la función y el deber social que tiene para con la comunidad y como él debe transmitir sus conocimientos de tal forma que sirvan para empoderar a la ciudadanía de ejercer sus derechos y que tanto como profesores y estudiantes vean en la pedagogía una forma de transformar el mundo, la sociedad y el saber (Villalobos, 2016) 


\section{Conclusiones}

En Colombia el proceso de la educación jurídica ha pasado por múltiples procesos históricos, los cuales han configurado de una u otra forma las dinámicas estatales en lo referido a la regulación normativa. Pero desde la década de 1950, diversos sectores han sentado una posición sobre el papel de la educación en el desarrollo social mediante la intervención de sus estudiantes, ya que la problemática radicaba en que no había una intervención de los mismos en los procesos de transformación que sufría el país.

Lo anterior no quiere decir que los estudiantes no ejercieran un papel activo dentro de la sociedad, pero los sectores críticos de los años 50 y 60 basaban sus críticas en estos aspectos. Por ello en la década de 1970 se da origen al Decreto 196 de 1971, que manifiesta primeramente la labor social del estudiante de Derecho en sus prácticas de Consultorio Jurídico. Las reformas educativas que se gestaron desde el año 1992 hasta el 2010, fueron destinadas a reformar aspectos administrativos de la misma, pasando luego por una modificación al Decreto 196 de 1971 con la Ley 583 de 2000, la cual trabajó en aspectos meramente procedimentales pero no sustanciales frente al rol social de los estudiantes de Derecho, lo que no significó un desarrollo sustancial en cuanto a la labor prestada por los Consultorios Jurídicos como prácticas jurídicas y su relación con la comunidad.

Con el panorama social que rodea al país, diferentes sectores del Estado y la academia han concordado que es necesaria una reforma estructural a la educación jurídica en Colombia, por lo que han salido a relucir conceptos como la enseñanza clínica del Derecho o las técnicas del litigio estratégico como una respuesta a la situación social de las facultades de Derecho. 
Ante esta necesidad, el objeto de este trabajo fue precisamente llegar a una propuesta estructural de práctica jurídica desde la figura del litigio estratégico, pero donde este cumpliera una función desde aspectos litigiosos pero a su vez pedagógicos que tengan como finalidad empoderar a las comunidades. Estos ejercicios demandan en sí mismos el incentivo de la investigación socio-jurídica que le permita al estudiante tener las herramientas teóricas y prácticas para trabajar con la comunidad.

Esto en concordancia a la necesidad de brindar alternativas que respondan a las críticas que pueda tener el ejercicio del Derecho como instrumento de dominación y mantenimiento del status quo, por intermedio del aparato normativo y su aplicación en el entorno social. Estas alternativas están dirigidas a visualizar el Derecho como un instrumento emancipatorio, que esté presente en los escenarios de discusión y permita, mediante practica y ejercicio alternativo del mismo, la reivindicación de derechos a esas comunidades vulnerables. Por ello, la figura del litigio estratégico puede ser tomada como una alternativa que responda a esas necesidades sociales y, a su vez, pueda ser una respuesta a las críticas que ha sido objeto el Derecho como ciencia occidental, desde el entendido que brinda un espacio al estudiante para que reflexione sobre la realidad social que lo rodea, desde espacios como la investigación o la interacción con la misma comunidad, y a su vez, le brinde la oportunidad al estudiante de proponer alternativas que permitan reivindicar derechos, exteriorizar o mitigar problemáticas.

Para tal fin, la labor pedagógica, la cual se debe realizar antes de iniciar con las prácticas que involucren de forma directa a la comunidad, debe adoptar los paradigmas de la pedagogía de la liberación, tal y como lo propone Freire, con el objeto de concientizar al estudiante que su labor contribuirá a la construcción de 
ciudadanías propias, desde la concepción abordada por Boaventura de Sousa Santos, en las que se fortalezca el empoderamiento de las comunidades que se han visto relegadas por las dinámicas sociales de la política y el conflicto, y se cree la conciencia, tanto en el estudiante como en la comunidad por intermedio del mismo, política y ética sobre el entorno y el territorio en el que se habita.

Esta dinámica contribuye a que el estudiante pueda determinar la función social que tiene su profesión de abogado y del compromiso que adquiere por esta razón, identificándose como un agente social de cambio. Trayendo como consecuencia que este mismo empoderamiento el estudiante lo transmita, a través de su accionar, a la comunidad con la que intercambia saberes, permitiendo cambiar y reformular, de manera paulatina, las dinámicas sociales que rodean su profesión y su entorno.

\section{Bibliografía}

Bocanegra, A. H. (2010). Las políticas educativas y el magisterio colombiano en la década de los 80. Dialogo de saberes, Vol. 1 Número 32. 29-44.

Bonilla, M. D. (2008). Igualdad, orientación sexual y derecho de interés público. La historia de la sentencia C-075/07. En C. Diversa, Parejas del mismo sexo: el cambio hacia la igualdad (págs. 11-40). Bogotá: Universidad de los Andes.

Carreño, M. T., \& González, C. V. (2017). Competencias investigativas versus competencias cientificas en la formación del abogado en Colombia. En A. C. (ACOFADE), Lineamientos técnicos 
para los programas de Derecho en Colombia (págs. 85-115). Medellín: Fondo editorial Corporación Universitaria Remington.

Castro, B. É. (2006). La enseñanza clínica: un paso hacia la calidad. Opinión jurídica, 175-186.

Cástro-Gómez, S. (2010). La Hybris del Punto Cero. Ciencia, raza e ilustración en la Nueva Granada (1750-1816) . Bogotá : Editorial Pontificia Universidad Javeriana.

Cazares, M. (9 de Septiembre de 2008). Mediateca Colombia aprende. Recuperado el 15 de Abril de 2018, de Colombia aprende: http://www.colombiaaprende.edu.co/html/mediateca/1607/ article-170863.html

Correa. M. L. (2008). Litigio de alto impacto: Estrategías alternativas para enseñar y ejercer el Derecho. Opinión Jurídica, 147-162.

De la Calle, M. C., \& Jiménez Armentia, P. (2011). Aproximación al concepto de responsabilidad social del universitario. Comunicación y hombre, 236-247.

Duque, Q. S., \& González, E. M. (2008). Los consultorios jurídicos y la popularización del Derecho. Prisma jurídico, 341-358.

Fals, B.00 O., Guzmán, C. G., \& Umaña, L. E. (1988). La violencia en Colombia. Bogotá: Circulo de lectores.

Gutierrez, G. C., \& Zarate, P. M. (11 de Marzo de 2017). Pensum histórico de la facultad de Derecho de la Pontificia Universidad Javeriana. Recuperado el 17 de Abril de 2018, de Slidex: https://slidex. tips/download/pensum-historico-de-la-facultad-de-derecho-dela-pontificia-universidad-javeriana

Helg, A. (1984). La educación en Colombia, 1918-1957: una his- 
toria social, económica y política. Bogotá: Universidad Pedagógica Nacional y Plaza y Janés.

Herrera, M. C. (1993). Historia de la educación en Colombia, la república liberal y la modernización de la educación: 1930-1946. Revista colombiana de educación N²6, 97-124.

Huertas, D. O. (2017). Conceptos fundamentales de la educación jurídica en Colombia. Una aproximación a los elementos curriculares en la enseñanza del Derecho. En A. C. (ACOFADE), Lineamientos técnicos para los programas de Derecho en Colombia (págs. 71-83). Medellín: Fondo editorial Corporación Universitaria Remington.

Londoño, T. B. (7 de Enero de 2016). Las Clínicas Jurídicas de interés público en Colombia retos y posibilidades de una naciente experiencia. Recuperado el 20 de Abril de 2018, de Biblioteca virtual. Centro de estudios de justicia de las Américas : http://biblioteca. cejamericas.org/bitstream/handle/2015/2644/paper_beatrizlondono.pdf?sequence $=1$ \&isAllowed $=y$

Ministerio de Justicia y del Derecho. (9 de Agosto de 2016). El Ministerio de Justicia trabaja para fortalecer los consultorios jurídicos, responder a las necesidades de los futuros abogados y mejorar el acceso a la justicia. Recuperado el 20 de Abril de 2018, de Minjusticia: http://www.minjusticia.gov.co/Noticias/Tabld/157/ArtMID/1271/ ArticleID/2429/El-Ministerio-de-Justicia-trabaja-para-fortalecerlos-consultorios-jur\%C3\%ADdicos-responder-a-las-necesidadesde-los-futuros-abogados-y-mejorar-el-acceso-a-la-justicia.aspx

Ministerio de Justicia y del Derecho. (Febrero de 2017). Proyecto de Ley de reforma a los Consultorios Jurídicos. Bogotá, Cundinamarca, Colombia.

Molina, B. C., \& González, C. V. (2017). Antecedentes de la for- 
mación jurídica en Colombia. En A. C. (ACOFADE), Lineamientos técnicos para los programas de Derecho en Colombia (págs. 15-31). Medellín: Fondo editorial Corporación Universitaria Remigton.

Montoya, E. M. (2017). La proyección social de la formación jurídica en el marco de la responsabilidad social universitaria. En A. C. (ACOFADE), Lineamientos técnicos para los programas de Derecho en Colombia (págs. 149-170). Medellín: Fondo editorial Corporación Universitaria Remington.

Probono. (s.f.). Análisis de clínicas jurídicas de facultades de abogacía. Recuperado el 20 de Abril de 2018, de Probono.org.ar: http://www.probono.org.ar/downloads/158_download.pdf

Ramírez, G. M., \& Téllez, C. J. (12 de Enero de 2006). La educación primaria y secundaria en Colombia en el siglo XX. Recuperado el 17 de Abril de 2018, de Banco de la República: http://www. banrep.gov.co/docum/ftp/borra379.pdf

Sentencia T-743 (Corte Constitucional 23 de Octubre de 2013).

Silva, R. A., Aguirre, R. J., \& Maldonado, J. F. (2013). Marx y la crítica de los derechos. Bucaramanga: Ediciones Universidad Industrial de Santander.

Villalobos, O. D. (2016). Ciudadanias propias. Una reflexión desde el diálogo entre Paulo Freire y Boaventura de Sousa Santos. Senderos Pedagógicos, 23-40. 